
\title{
R Research Square \\ COVID-19 in the peritoneal fluid: does this evidence oblige to introduce new rules? Presentation of a Case Report
}

Edoardo Rimini ( $\nabla$ edoardorimini@libero.it )

Ospedale Policlinico San Martino

Giulia Atzori

Ospedale Policlinico San Martino https://orcid.org/0000-0001-9206-9432

Alessandro Viotti

Ospedale Policlinico San Martino

Case Report

Keywords: Covid, surgery, peritoneal swab, case report

Posted Date: May 12th, 2020

DOI: https://doi.org/10.21203/rs.3.rs-28579/v1

License: (1) (i) This work is licensed under a Creative Commons Attribution 4.0 International License.

Read Full License 


\section{Abstract}

Background : The Covid 19 pandemic introduced the need to outline new guidelines for the treatment of the Covid positive patient in surgery. With this report we present what we believe to be important additional information about an area that has not yet been studied, the potential infectivity of peritoneal secretions.

Case presentation : A 73-year-old woman accesses our PS where she is found to be positive on the nosepharyngeal swab for Covid 19. The patient presents a picture of intestinal occlusion from imprisoned hernia, which is why she undergoes exploratory laparotomy surgery. During surgery a peritoneal swab is performed which is positive for Covid 19.

Conclusion : This case suggests that, in the presence of patients susceptible to surgical therapy with negative Covid swab, performing a peritoneal swab may reveal a false negative. An extensive database that collects the results of pharyngeal and peritoneal swabs of patients undergoing surgery is necessary to delineate the appropriate approach to the patient in the Covid era.

\section{Background}

The COVID-19 pandemic forced the rapid introduction of new guidelines directed to doctors, nurses, and health-care workers in general, while performing clinical practices, in order to allow the treatment of COVID patients preserving the personnel from infection. The surgical environment has also been significantly affected by new rules aimed at reducing as much as possible the possibility of virus exposure to surgeons, nurses, and all the health-care workers in the operative room.

Guidelines have been quickly introduced by most international surgical societies, to give the most consistent instructions while facing surgical cases in patients at-risk or frankly positive for COVID-19, particularly in an emergency setting. ${ }^{(1)}$

We believe that this report might give important additional information on a topic that, to our knowledge, has not been covered yet: that of the potential infectiousness of peritoneal secretions.

\section{Case Presentation}

The patient, a 73 year-old woman, came to our E. R. the 06/04/2020 for acute abdominal pain in the central abdomen associated to a painful bulge in the umbilical area, in the absence of fever.

The physical examination revealed that the periombilical lump was hyperemic, hard, fixed, and nonreducible. Blood tests revealed leukocytosis (15.300) and an elevated C-reactive protein (356). As indicated in our current protocol, at hospitalization she also immediately underwent nasopharyngeal swab and specific immunoglobin $\mathrm{M}(\mathrm{IgM})$ and immunoglobulin $\mathrm{G}(\mathrm{IgG})$ screening testing, all of them resulting positive for Covid-19 infection. 
She then underwent total-body CT scan that did not reveal any sign of active pneumonia, but showed a large umbilical hernia in which some loops of the small intestine were engaged, with stagnation of liquid and some air-fluid levels. The jejunal loops were thickened and hyperemic, and presented edematous imbibition of perivisceral soft tissues. There was also the evidence of free fluid in the pelvis.

Emergency surgery was therefore indicated, and the patient prepared for surgery, despite the recent reports of a poor prognosis in Covid-19 positive patients undergoing surgery. ${ }^{(2)}$ Surgery was performed following all our current protocols for Covid-19 positive patients and consisted of an explorative laparotomy that immediately confirmed the presence of free fluid in the abdomen and the presence of a strangled hernia with incarcerated and necrotic jejunal loops that did not show any sign of perforation. A sampling of peritoneal fluid was collected at the beginning of the procedure and sent for Covid-19 testing. Surgery then consisted of an ileal resection with immediate latero-lateral anastomosis, and the suture of the hernial defect with separate stitches.

The postoperative course was uneventful and the patient is currently hospitalized in the infectious disease department of our hospital.

Forty-eight hours after surgery the result of the peritoneal fluid sampling was obtained, and resulted positive for Covid-19.

\section{Discussion}

The evidence of the presence of Covid-19 in the peritoneum opens a new scenario about the necessity of further guidelines to adopt while operating in the Covid-19 pandemic era, since the presumed safer nature of a traditional open surgery over the laparoscopic technique, with regard to surgeons, nurses, and the surgical environment, should be reassessed.

With this evidence, we should introduce a word of caution with respect to those cases undergoing emergency surgery who are negative at nasopharyngeal swab only, a procedure that is currently not entirely reliable for the diagnosis of Covid-19 infection. ${ }^{(3,4)}$ In this setting, if the patient has a false negative test, the possibility of infection to health-care workers in the operative room is significant, if guidelines for Covid-19 positive patients are not followed.

In the clinical practice, due to the actual absence of strong evidences on the diffusion of Covid-19, this report allowed us to conclude that all patients undergoing emergency surgery should be considered Covid-19 positive, despite a negative results at common testing.

In consideration of the result obtained in this case, a protocol that involves both pharyngeal and peritoneal swabs has been implemented for patients undergoing emergency surgery. Further data will be analysed in the future thanks to the database that we are developing.

\section{Abbreviations}


E. R.: emergency room

\section{Declarations}

Ethics approval and consent to participate : The authors declare that they have obtained written consent to the processing of personal data for the aims of the study.

Consent for publication : The authors declare that they have obtained written consent to the publication of the participant's data. Consent is available from the corresponding author on request.

Availability of data and materials : The datasets used and/or analysed during the current study are available from the corresponding author on reasonable request

Competing interests : The authors declare that they have no competing interests

Funding : The authors declare that they have not received funding

Authors' contributions : Study conception and design: E.R.; Acquisition and interpretation of data:E.R, A.V.; Drafting and revision of manuscript : E.R., G.A. All authors read and approved the final manuscript

Acknowledgements : not applicable

\section{References}

1. Coccolini F, Perrone G, Chiarugi M et al. Surgery in COVID-19 patients: operational directives. World J Emerg Surg. 2020 Apr 7;15(1):25. doi: 10.1186/s13017-020-00307-2

2. Mi B, Chen L, Xiong Y et al. Characteristics and Early Prognosis of COVID-19 Infection in Fracture Patients. J Bone Joint Surg Am. 2020 Apr 1. doi: 10.2106/JBJS.20.00390

3. Kokkinakis I, Selby K, Favrat B et al. Covid-19 diagnosis : clinical recommendations and performance of nasopharyngeal swab-PCR. Rev Med Suisse. 2020 Apr 8; doi: 16(689):699-701

4. Winichakoon P, Chaiwarith R, Liwsrisakun $\mathrm{C}$ et al. Negative Nasopharyngeal and Oropharyngeal Swab Does Not Rule Out COVID-19. Q. J Clin Microbiol. 2020 Feb 26. pii: JCM.00297-20. doi:

10.1128/JCM.00297-20

\section{Figures}




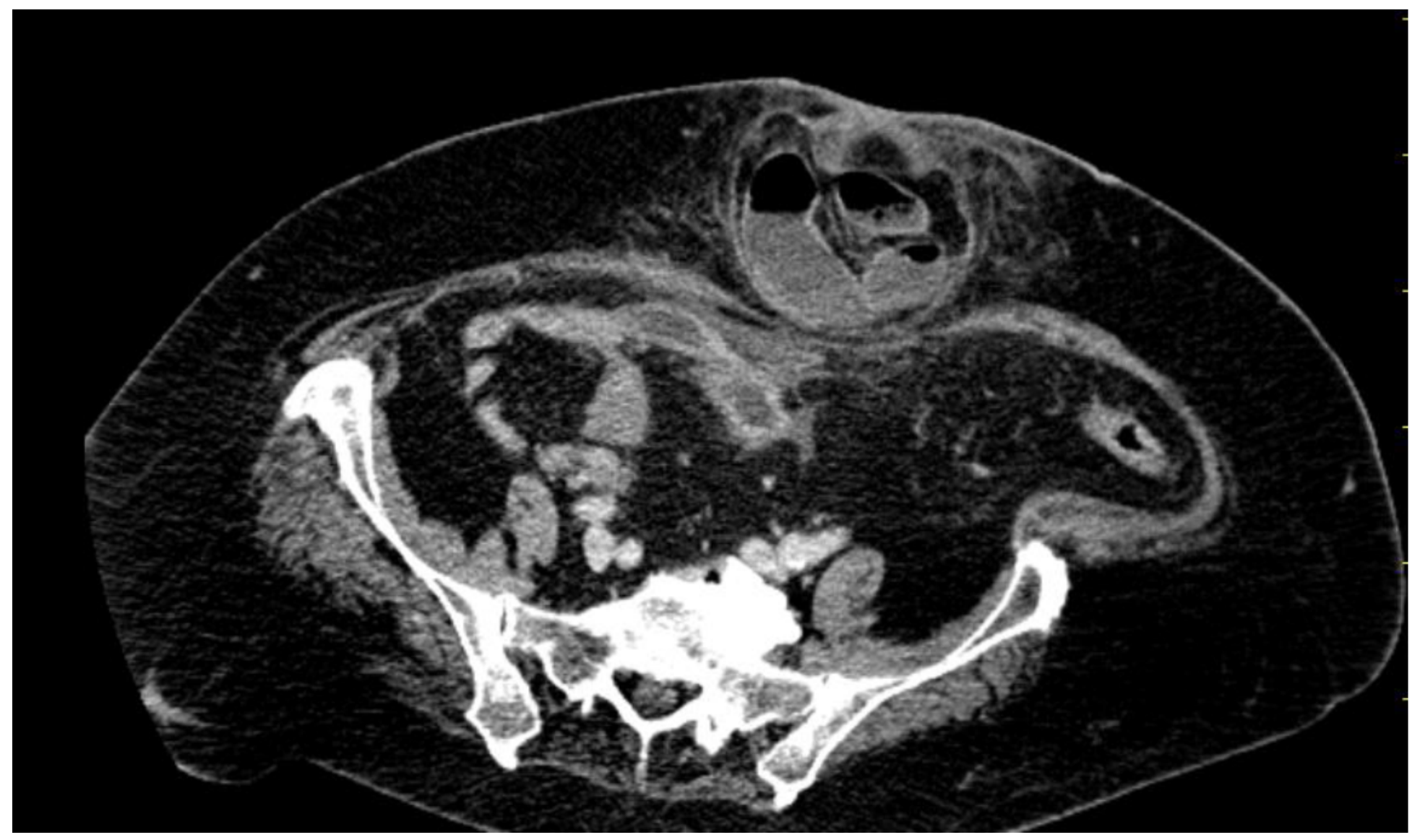

Figure 1

Abdomen CT-scan 